\title{
A situação da atenção farmacêutica: revolução ou penumbra paradigmática?
}

\author{
The situation of pharmaceutical care: revolution or paradigmatic \\ twilight?
}

Thais Penaforte', Sabrina Castro ${ }^{2}$

DOI: 10.1590/0103-1104202113108

RESUMO Com o propósito de compreender a situação da atenção farmacêutica em um sistema municipal de saúde, tomou-se como ponto de partida o estudo do grau de adesão e incorporação do paradigma profissional que orienta essa prática. Com um desenho qualitativo e de abordagem etnográfica, os critérios que orientaram essa investigação buscaram tipificar a variedade de cenários, motivações e procedimentos que se associam a esse exercício profissional. Esse recorte permitiu examinar se os movimentos que operam a atenção farmacêutica ainda constituem uma área de penumbra ou se já foi alcançada a concretude de um novo modelo de cuidado para a farmácia. Os resultados informam uma variedade de arranjos, desenhos construtivos e estrutura conceitual. Não há congruência nem na delimitação de seus componentes estruturantes. Todo esse cenário revela que ainda se está em uma fase de penumbra ou 'crise paradigmática', na qual o antigo paradigma ainda não foi totalmente abandonado ante a superioridade do novo. Isso se observa na ausência de uma interpretação padronizada capaz de revelar elementos recorrentes e compartilhados. Desse modo, o reconhecimento de uma realização exemplar, capaz de consolidar a maturidade e transição paradigmática da atenção farmacêutica, não foi encontrado.

PALAVRAS-CHAVE Farmacêuticos. Assistência farmacêutica. Sistema Único de Saúde. Relações profissional-paciente. Farmácia.

ABSTRACT In order to understand the situation of pharmaceutical care in a municipal health system, we took as a starting point the study of the degree of adherence and incorporation of the professional paradigm that guides this practice. With a qualitative design and an ethnographic approach, the criteria that guided this investigation sought to typify the variety of scenarios, motivations and procedures that are associated with this professional exercise. This cutout allowed us to examine whether the movements that operate pharmaceutical care are still an area of darkness or the concreteness of a new care model for Pharmacy has already been achieved. The results inform a variety of arrangements, constructive designs, and a conceptual framework. There is no congruence or delimitation of its structuring components. The entire scenario reveals that we are still in a phase of twilight or 'paradigmatic crisis', in which the old paradigm has not yet been totally abandoned in the face of the superiority of the new one. This is observed in the absence of a standardized interpretation capable of revealing recurrent and shared elements. Thus, the recognition of an exemplary achievement, capable of consolidating the maturity and paradigmatic transition of pharmaceutical care, was not found.

1 Universidade Federal da Bahia (UFBA) - Salvador (BA), Brasil.

thaisrpenaforte@gmail.com

2 Universidade Federal do Oeste da Bahia (Ufob) Salvador (BA), Brasil.
KEYWORDS Pharmacists. Pharmaceutical services. Unified Health System. Professional-patient relations. Pharmacy. 


\section{Introdução}

A definição do conjunto de atitudes e valores específicos à determinada classe profissional é um desafio a quase todas as profissões. Em vista disso, esse campo é repleto de tensões e disputas dada a natureza complementar das práticas profissionais em saúde. No cenário da farmácia, essa realidade se reproduz a partir de inúmeras situações e agentes, que contribuem para uma espécie de conflito ontológico latente. Atrelado a isso, as inúmeras transformações que sofreram os serviços farmacêuticos alteraram significativamente o curso de suas atividades e, especialmente, a atuação desse profissional.

Os boticários, com sua prática notadamente voltada para a arte das formulações terapêuticas e forte ênfase nos conhecimentos farmacológico e químico medicinal, lançaram as bases para o futuro exercício profissional farmacêutico'. Com a criação dos primeiros Cursos de Farmácia, observou-se também as primeiras mudanças no seu modelo de atuação. Da tradição artesanal para o adensamento do processo industrializante, o farmacêutico refinou seu talento técnico ao tempo em que passou a acompanhar todas as transformações do setor saúde. Com a consolidação do Sistema Único de Saúde (SUS) e as exigências de uma assistência integral, contínua e que inclua as dimensões singulares e subjetivas do processo terapêutico, o farmacêutico viu-se, mais uma vez, desbravando novos núcleos de atuação. Essas transformações sedimentaram novas práticas ao seu ofício, que busca acompanhar e se adaptar às demandas sanitárias, econômicas e da própria sociedade.

Esta constante exigência, de se expandir a diferentes papéis e modelos assistenciais, conforma um campo controverso, em que o novo e o antigo estão sempre confrontando interesses e gerenciando a mudança. Desse modo, seria ingênuo supor que, sobre a prática farmacêutica, suas definições e arcabouço político, implicaria um discurso monolítico de seu domínio e conteúdo. Ao contrário, os farmacêuticos delimitam seu princípio de ação não de uma maneira estática, mas, incluem a si mesmos e suas experiências como conteúdo propositivo. Não à toa, o trabalho de Costa e colaboradores ${ }^{2}$ revelou a dificuldade em delimitar um marco teórico-conceitual para a assistência farmacêutica. Como solução, para os autores, o conteúdo da assistência farmacêutica "deve ser o resultado da combinação de estrutura, pessoas e tecnologias, num dado contexto social"2(2).

No que tange à oferta de atividades de natureza clínica, Araújo e colaboradores ${ }^{3}$ demonstraram como as dificuldades para a caracterização dessas atividades e compreensão de seu significado decorrem de um cenário de incipiente institucionalização e fragilidade, na definição dessas práticas pela Política de Assistência Farmacêutica. Além disso, destacam um cenário de "insuficiente discussão conceitual e filosófica no país sobre a atenção farmacêutica e as próprias disputas do campo científico"3(9).

Considerada o mais recente desafio para os farmacêuticos, a atenção farmacêutica reorienta o foco de atenção e da prática profissional, estimulando uma relação cooperativa entre farmacêutico e paciente, com vistas ao atendimento de todas as suas necessidades terapêuticas. Em 1990, Hepler e Strand 4 instigaram os farmacêuticos a adotar um novo paradigma profissional, com uma atuação centrada no paciente e orientada para resultados. Dessa forma, a definição dos autores para pharmaceutical care qualifica-se como uma proposta de transformação laboral e filosófica, que aciona e tensiona definições de autoridade, objeto, modos de ação e finalidade da ação farmacêutica.

Outra proposta estruturante para a atenção farmacêutica, o Método Dáder, caracteriza-se como um procedimento operativo para o acompanhamento da farmacoterapia em qualquer nível de cuidado e para qualquer paciente. A aplicação dessa metodologia visa criar alguns padrões de prática, que garantam a eficiência do serviço e, acima de tudo, a segurança do paciente 5 . 
A constituição brasileira de um arcabouço político para a atenção farmacêutica foi inaugurada com a inclusão dessa prática na Política Nacional de Assistência Farmacêutica (PNAF) ${ }^{6}$. Posteriormente, o Ministério da Saúde brasileiro, ao divulgar um caderno de orientação para os serviços farmacêuticos na atenção básica, propôs, como estratégia de integração da assistência farmacêutica às Redes de Atenção à Saúde (RAS), um novo modelo assistencial - o cuidado farmacêutico. Reconfigurado a partir de uma maior ênfase na atuação clínica dos farmacêuticos, esse modelo de ação é teorizado e organizado a partir da própria estrutura da atenção básica, apoiando-se nas duas dimensões do apoio matricial: a clínico-assistencial e a técnico-pedagógica ${ }^{7}$.

É possível observar que apreensão brasileira da definição de atenção farmacêutica abriga as disposições filosóficas do pharmaceutical care de Hepler e Strand ${ }^{4}$, a estrutura metodológica do Método Dáder ${ }^{5}$ e, também, um tipo de delineamento experimental a partir das RAS ${ }^{7}$. Essa diversidade de disposições, visões e conceitos elabora um arranjo nacional variado, que ora opta pelo termo atenção farmacêutica ${ }^{6}$, ora cuidado farmacêutico 7 .

É possível observar que, a despeito da variabilidade nas configurações teóricas e metodológicas, tanto nas definições de atenção farmacêutica quanto de cuidado farmacêutico, é explícita a intenção de instituir ao trabalho farmacêutico um novo paradigma profissional - centrado no cuidado ao paciente. Nesse sentido, uma compreensão mais aprofundada desse processo evolutivo exige que se analise o grau de expansão desse paradigma no cotidiano farmacêutico. Assim, nesta investigação, interessa compreender em que extensão os farmacêuticos atuantes em um sistema municipal de saúde aderem e formulam esse novo paradigma profissional. Dessa forma, a essa questão principal, pretende-se adensar outras reflexões, dirigidas à própria natureza conceitual da atenção farmacêutica, seus elementos constituintes e configurações projetadas pelos farmacêuticos.

\section{Metodologia}

A fim de analisar as perspectivas compreensivas e as escalas paradigmáticas do exercício da atenção farmacêutica, optou-se por uma pesquisa qualitativa de abordagem etnográfica. Ao pretender descrever e interpretar a arquitetura e a aplicação conceitual dessa prática profissional, buscou-se examinar as relações sociais, profissionais e institucionais que dirigem os serviços farmacêuticos.

A tipificação de um corpo ontológico e metodológico à atenção farmacêutica parte do postulado de que o enfoque centrado no paciente é, propriamente, a filosofia e o alicerce paradigmático desse exercício profissional. Assim, adotou-se como definição de paradigma a proposta de Thomas Kuhn ${ }^{\mathbf{8 ( 2 1 8 )}}$ como "toda constelação de crenças, valores, técnicas, etc..., partilhadas pelos membros de uma comunidade determinada" e que "durante algum tempo, fornecem problemas e soluções modelares para uma comunidade de praticantes $[. .] " .8(13)$.

Para Masterman', o paradigma kuhniano é um artefato ou uma entidade, que, de um ponto de vista mais instrumental e concreto, organiza 'um modo de ver' do qual depende todo êxito e método das realizações científicas. Ainda na visão de $\mathrm{Kuhn}^{\mathbf{8 ( 2 2 4 )}}$, é o próprio paradigma que define as lentes e os operadores dos novos campos da experiência. Desse modo, "um paradigma governa, em primeiro lugar, não um objeto de estudo, mas um grupo de praticantes [...]".

Os critérios que orientaram essa investigação buscaram tipificar a variedade de cenários, motivações e procedimentos que se associam ao ofício farmacêutico e sua situação paradigmática. Esse desenho permitiu examinar se os movimentos que operam a atenção farmacêutica ainda constituem uma área de penumbra (ocupada por realizações cujo status ainda é duvidoso) ou se já foi alcançada a concretude de um novo modelo de cuidado para a farmácia. 


\section{Cenário e interlocutores}

O município estudado possui uma rede de atenção primária à saúde subdividida em 12 distritos sanitários. Ao longo do trabalho de campo e tendo percorrido seis distritos, observou-se que os resultados começaram a se repetir de forma muito contundente, o que sugeriu a saturação dos resultados e a inexistência de alterações significativas nos demais distritos. Assim, esta investigação apresenta o resultado de 36 unidades de saúde visitadas (do total de 64 unidades existentes). Destas, 25 eram unidades básicas de saúde, 3 centros de atenção psicossocial e 8 unidades da saúde da família.

Do total de unidades visitadas, em apenas 13 encontraram-se farmacêuticos presentes. Em 17, havia um responsável pelo atendimento (não farmacêutico); e em 6, não foi possível o acesso às informações (entrevista declinada).

Durante a pesquisa, buscou-se lidar tanto com a base conceitual quanto vivencial dos interlocutores, investigando as composições de ideias que constituem seus próprios emaranhados atitudinais. Assim, o conjunto metodológico deste trabalho se orientou pelo retrato empírico dos estabelecimentos farmacêuticos, a partir da observação participante e aplicação de entrevistas semiestruturadas aos farmacêuticos. Em cada unidade de saúde, buscou-se identificar as cenas de ação e trocas com os usuários, que forneceram pistas sobre os contornos das concepções de atenção farmacêutica performadas, assim como as condições de funcionamento dos serviços. Tanto a observação participante quanto as entrevistas semiestruturadas foram orientadas por um roteiro sistematizado a partir de duas dimensões:

Dimensão atitudinal-profissional - contemplou questões relativas às características formativas, de ascensão ao serviço público de saúde e as experiências vividas com a atenção farmacêutica. Foram observadas as condições dessa trajetória e vivência profissional, assim como suas possíveis implicações na atuação profissional.

Dimensão conceitual - objetivou capturar a concepção acerca da atenção farmacêutica e seus contornos conceituais. Contemplou também a observação da aplicabilidade desse corpo de conhecimentos à rotina assistencial.

Para a interpretação dos dados obtidos, utilizou-se a análise de discurso ${ }^{10}$ como estratégia analítica, adotando os conceitos oficiais como metáforas representacionais. Todo material empírico foi analisado utilizando-se o software Atlas $\mathrm{ti}^{\circledR}$, que contribuiu para composição e exploração dos dados. O processo analítico envolveu a transcrição detalhada das entrevistas seguida da exploração do material, aplicando o espírito da leitura cética proposto por Gill10. Nesse tipo de procedimento, o analista direciona o olhar à construção, à organização e às funções do discurso ao invés de buscar algo subjacente a ele. Esse momento exigiu um interesse não na busca de essências ou sínteses dos relatos, mas nos detalhes das passagens e relações empreendidas e nas confluências entre aquilo que foi dito e sua interseção com a realidade observada. Em seguida, procedeu-se à etapa de codificação, determinada a partir de categorias suscitadas pelos discursos dos farmacêuticos e os contornos informados à atenção farmacêutica.

O processo de interpretação buscou emergir a existência de fatores norteadores à consolidação da atenção farmacêutica e seu paradigma profissional. Portanto, essa prática foi analisada a partir de sua congruência às novas categorias e procedimentos paradigmáticos. Tendo ocorrido de maneira transversal ao estudo, a elucidação tanto da mediação técnica quanto intelectual que operaram a atenção farmacêutica levou em consideração os elementos observados nas entrevistas, a vivência dos cenários de pesquisa e o arcabouço teórico vigente nas políticas de assistência farmacêutica.

Este trabalho, além de ser desenvolvido sob os mais elevados princípios éticos, foi aprovado pelo Comitê de Ética em Pesquisa da Escola de Enfermagem da Universidade Federal da Bahia (CAAE: 01288818.3.0000.8035). Todos os participantes assinaram o Termo de Consentimento Livre e Esclarecido. 


\section{Resultados}

\section{Relação entre componentes do trabalho farmacêutico, trajetórias e atitudes dos farmacêuticos}

Observou-se que, de maneira geral, o grupo de farmacêuticos estudados apresentou uma trajetória profissional fracamente direcionada à assistência farmacêutica. Apenas três farmacêuticos indicaram ter formação continuada ou identificação profissional específica para esse campo. Quando questionados acerca de sua área de atuação preferencial, a mais citada foi a de análises clínicas $(69,2 \%, 9 / 13)$.

Quanto à motivação para ingresso na Secretaria Municipal de Saúde, parte dos farmacêuticos revelou ter optado por trabalhar na assistência farmacêutica básica por uma questão de oportunidade e facilidade, incentivado, muitas vezes, pelo tipo de vínculo empregatício e pela baixa concorrência do campo:

Dos quinze profissionais, os treze queriam farmácia bioquímica eu fui a última a ser entrevistada [...] eu disse: farmácia, eu quero farmácia. Pela concorrência, quando eu entrei foi pela concorrência eu não tinha experiência. (Farmacêutico 1).

Ahé eu me formei em análises clínicas, mas eu nunca atuei, então eu vim para cá. (Farmacêutico 6).

Fiz concurso público porque é um concurso público, não porque eu queria trabalhar, né, não exatamente. (Farmacêutico 7).

Para outro grupo de profissionais, a opção pelo campo a assistência farmacêutica decorre de uma identificação e vinculação com área e seu escopo de atuação:

Existiam duas vagas para farmacêutico bioquímico $[$ [...] todo mundo querendo ir para o laboratório [...] foi muito bom a minha escolha, não me arrependo a agradeço à vida ter me dado essa oportunidade [...] hoje eu sou extremamente realizada. (Farmacêutico 1).
Eu gostava de bioquímica [...] mas, quando eu ingressei na área de farmácia, né, na parte pública, né, é apaixonante porque você vê a necessidade do profissional farmacêutico. (Farmacêutico 7).

A fim de caracterizar o trabalho assistencial realizado pelos farmacêuticos nas unidades de saúde e instrumentalizados pelas observações de campo, alcançou-se a identificação de um conjunto de quatro grupos de atividades, que foram descritas pelos profissionais como constituintes de sua rotina de trabalho: 1) atividades de assistência ao paciente; 2) supervisão da equipe; 3) planejamento da assistência; e 4) atividades administrativas e de gerenciamento.

Quando estimulados a indicar quais grupos de atividades concentravam o maior volume de trabalho dedicado, observou-se que as atividades administrativas e de gerenciamento; a supervisão da equipe e o planejamento da assistência ocupavam o maior espaço da rotina profissional para $84,6 \%(11 / 13)$ dos farmacêuticos. Apenas para 15,4\% (2/13) a atividade mais realizada se relacionava com as ações de assistência ao paciente.

A experiência etnográfica nas farmácias confirmou o diagnóstico realizado pelos farmacêuticos. O olhar sobre o cotidiano das práticas assistenciais permitiu evidenciar um cenário em que imperava um tipo de exercício farmacêutico convencional, limitado à distribuição de medicamentos e com pouco alcance ao cuidado direcionado ao paciente. A presença do farmacêutico no momento da dispensação ocorria de maneira bastante irregular e mais comumente restrita a episódios 'problemáticos' que exigiam a presença do profissional para juízo da situação. A agência do farmacêutico em redefinir a situação assistencial é fracamente percebida; e mesmo nos poucos encontros clínicos, sua atuação parece mais perscrutar sinais de desvio ou 'desobediência' ao regime prescrito do que estabelecer vínculo ou objetivos terapêuticos.

No entanto, no contexto desse aparente imobilismo assistencial, pudemos observar alguns conflitos internos entre os farmacêuticos e 
sua atuação profissional. Suas opiniões sobre a postura da administração municipal, da comunidade assistida e da própria academia constroem uma trama de frustrações, descontentamentos e afastamentos, que afetavam sua capacidade de ação e de transformação da realidade. $O$ desejo de uma atuação mais clínica e humanizada não é esquecido por parte significativa desses profissionais, mas é bastante dificultado, segundo eles, tanto por questões estruturais quanto operacionais.

\section{Dimensão conceitual, aspectos construtivos e a prática da atenção farmacêutica}

Para alguns farmacêuticos, o conteúdo da atenção farmacêutica segue predominantemente os fundamentos teóricos da assistência farmacêutica, acrescida da ampliação do foco de trabalho para o paciente, como exemplifica o seguinte trecho de uma das entrevistas:

\begin{abstract}
Atenção farmacêutica significa, acho, um conjunto de ações que vai desde o planejamento da aquisição do medicamento e, até você chegar ao paciente acho que a atividade de atenção farmacêutica engloba tudo que vocêfaz é pensando no paciente tanto na qualidade do que você adquire, quanto a confiabilidade dos produtos que você manipula até chegar ao paciente, até chegar não, talvez tudo isso o objetivo principal é o paciente, porque você tem um planejamento na sua compra, na sua aquisição. Você tá fazendo isso tudo por quê? Porque você tem a causa inicial de tudo isso, que movimenta tudo, que é o paciente. (Farmacêutico 10).
\end{abstract}

Em sentido oposto aos conceitos oficiais, verificou-se que, para alguns profissionais, a atenção farmacêutica não é em si uma prática exclusiva do farmacêutico, mas se caracteriza como um serviço multiprofissional:

Atenção farmacêutica é um conjunto de atividades que o farmacêutico presta juntamente com outros profissionais de saúde pra garantir o uso racional de medicamentos. (Farmacêutico 5).
Grande parte da abordagem acadêmico- científica relacionada com a atenção farmacêutica se reporta ao conceito de saúde em sua dimensão ampliada. No entanto, muitos farmacêuticos não conseguem extrapolar o cuidado para além do medicamento:

A atenção farmacêutica é você acompanhar o uso de medicamentos pelo paciente, né, na evolução mesmo da cura dele ou do tratamento, se aquela medicação que você tá dispensando e que foi prescrita pelo médico, se ela está realmente adequada para aquele tipo de patologia que o paciente tem, se não tem erro, se o tratamento é o correto. (Farmacêutico 4).

Então a gente poderia pelo menos tentar acompanhar esse paciente, o uso dele, o uso desse medicamento, ver efeitos colaterais de medicamentos, ver ação realmente do medicamento, ver se aquele medicamento tá tendo a ação esperada. (Farmacêutico 13).

A atenção farmacêutica pra mim seria é, não fazer uma entrega pura e simplesmente dos medicamentos. Seria explicar ver como a o que é que o paciente está tomando, né. Porque a grande maioria toma, né, o medicamento de forma errada, para de tomar por conta própria. A população ela precisa muito, ela pede por, carece do, do profissional pra tomar conta, né, foi aquele, guardião realmente dos medicamentos. (Farmacêutico 9).

Entretanto, alguns profissionais apresentam um olhar ampliado sobre o cuidado, identificando outras dimensões terapêuticas e ultrapassando a perspectiva medicamento-doença:

Acho que o farmacêutico tem que sair, participar, verificar, ver as dificuldades com relação ao paciente, com relação ao familiar, ver a questão que transcende essa parte aqui, e ir pra convivência dele, né, no dia a dia. Como é que é visto ele, né? O que a doença significa pra ele, né? Que estigma ele traz, né? Uma série de coisas que você precisa primeiro entender, né, a dinâmica do paciente, pra depois você poder atuar enquanto profissional. (Farmacêutico 2). 
A orientação dos conceitos oficiais é bastante propositiva à retomada da função clínica e social do farmacêutico. Contudo, os desafios práticos inerentes a esse tipo de atuação, notadamente inclinada ao atendimento individual, ocasionam certa apreensão:

É difícil realizar na prática. A gente faz pouquíssimo, muito pouco, às vezes a gente dá uma orientação ao paciente ali na hora que ele vai pegar o medicamento porque as condições de trabalho, primeiro, a gente tem um fluxo grande de pacientes, depois o local de trabalho não favorece você atender, eu não consigo fazer o que a universidade propõe. (Farmacêutico 3).

Em uma tentativa de encontrar uma estrutura de conteúdo, para a atenção farmacêutica, a partir da livre definição pelos farmacêuticos, as mais variadas combinações foram reproduzidas. Essa variedade de arranjos dimensiona a abrangência de significados, elementos e correlações que essa prática suscita. As categorias mais destacadas são descritas a seguir:

a) Acompanhamento farmacoterapêutico

Fazer esse tipo de acompanhamento [terapêutico], pelo menos isso seria o mínimo de uma atenção farmacêutica. (Farmacêutico 3).

\section{b) Dispensação e orientação}

Bom, além da, dispensação de medicamentos, a parte mais importante que eu acho é a orientação mesmo ao paciente, né, tá acompanhando se ele está realmente é... tomando aquele medicamento correto, de acordo com a prescrição do médico, se ele tem alguma queixa com aquele medicamento, se ele tá causando algum, algum efeito colateral. (Farmacêutico 12).

\section{c) Intervenção farmacêutica}

Eu acho que na atenção envolve primeiro a orientação, o acompanhamento farmacoterapêutico, né, e depois é muito importante as intervenções que a gente possa vir a fazer em termos de dosagem ou de mudança de prescrição. (Farmacêutico 6). d) Entrevista farmacêutica

Eu acho que a primeira atividade da atenção farmacêutica que o profissional tem que aprender a saber lidar é uma entrevista então, saber fazer essa escuta, e saber é, fazer uma entrevista é o primeiro passo pra você poder ajudar o outro. (Farmacêutico 2).

Uma dúvida recorrentemente apresentada pelos farmacêuticos foi eminentemente ontológica. Sendo de composição plural, quando o exercício profissional atende apenas parcialmente à composição referente, pode-se caracterizá-la ou não como atenção farmacêutica? Qual seu elemento mestre, a metodologia ou a adesão ao paradigma filosófico?

Bom, aqui, assim, a gente não trabalha assim, diretamente com essa atenção farmacêutica, mas eu como profissional, eu me preocupo, né, com os pacientes tem paciente que as vezes chega na farmácia com queixa de efeitos colaterais, e me pergunta até se ele pode mudar o medicamento, ai, ai eu vou conversar com o médico, digo que o paciente $x$ está sentindo, e se ele pode mudar esse medicamento. Então assim, eu como profissional aqui de farmácia, e como me dedico, eu me preocupo, mas não se posso dizer que faço. (Farmacêutico 4).

Para alguns eu digo que faço atenção farmacêutica, porque me preocupo, faço acompanhamento dos pacientes, mas para outras pessoas como eu não faço registro então eu não faço, então depende de quem eu estou falando. (Farmacêutico 12).

Dentro da minha visão, do que eu vejo como atenção farmacêutica, eu tenho, faço grupo com família, nesse grupo de família a gente trata do tema de medicamento, né, as dúvidas com relação a ele, as dúvidas com relação à doença, né, o que é pra família ter o paciente doente, como é dar a medicação [...]. Então esclarecer todas as coisas, efeitos colaterais de medicamento. (Farmacêutico 2). 
A atenção farmacêutica aqui é mínima, quando o paciente solicita a minha atenção e, as vezes eu, quando eu aproveito o momento da fila grande, da espera do paciente, que o paciente vai ter que esperar, e aí eu vou conversando com cada um e tirando dúvida. [...] Tem o pouco que eu ainda posso oferecer, a gente faz para não fazer nada, melhor do que não fazer nada, fazer alguma coisa, entendeu? Mas não como deveria, como a gente aprende na faculdade, mas o que a gente tem. (Farmacêutico 1).

Em nenhuma das farmácias analisadas foi possível encontrar elementos estruturais que garantissem condições mínimas para o exercício da atenção farmacêutica. Em apenas duas unidades era realizado algum tipo de atendimento estruturado, direcionado ao paciente.

Em uma delas, todos os pacientes eram atendidos individualmente, em um ambiente que, a despeito de seu desenho tradicional de 'janelinha de atendimento', a farmacêutica optou por inutilizar esse espaço e acolhia os pacientes pela única porta de entrada da farmácia, compartilhando sua mesa de trabalho para apoio à dispensação e desenvolvimento de ações de cuidado específicas. Tratava-se de uma unidade de atenção psicossocial, e o seu baixo volume de atendimento favorecia a possibilidade de concretização desse rearranjo interno. No entanto, esse novo desenho exigia uma reorganização administrativa, uma vez que essa sobreposição de espaços, algumas vezes, gerava comprometimentos - por exemplo, nos momentos de recebimento de medicamentos, os atendimentos tinham que ser suspensos. Além disso, havia uma constante interrupção do serviço farmacêutico pelos pacientes que aguardavam o seu atendimento ou demandavam informações pontuais.

Em outra unidade de saúde, cujo desenho assistencial era notadamente voltado para ações de base comunitária, o ambiente da farmácia (como resultado da ação política e envolvimento da farmacêutica) alcançou um desenho arquitetônico que favorecia as ações de dispensação e orientação individualizada, com cadeiras para atendimento e fila de espera organizada. O espaço de interação era envidraçado, mas ainda preservava uma maior visualização do ambiente e do atendente, com menor ruído à comunicação.

Nas outras 11 unidades, em que foram encontrados farmacêuticos presentes, os ambientes possuíam um desenho precário às ações que se propunham realizar. Os espaços de atendimento limitavam-se a pequenas aberturas nas paredes ou 'janelas de atendimento', muitas vezes com adicional de grades, e que, sendo, por vezes, tão diminutas, dificultavam qualquer tipo de visualização entre os indivíduos. A comunicação nessas situações era tão comprometida que era difícil identificar se ambas as partes conseguiam compreender um ao outro.

A irregularidade no abastecimento de medicamentos era, também, uma circunstância crônica segundo os interlocutores. A insuficiência desses insumos pôde ser claramente observada durante o período de estudo. Muitas vezes, o reabastecimento dos medicamentos faltosos era insuficiente para a cobertura da demanda, e a recomposição dos estoques podia não ocorrer por mais de seis meses.

Esse tipo de estrutura debilita não apenas o desempenho das ações de saúde, mas também a própria conexão entre o serviço e os pacientes. Estabelece-se uma relação pouco amistosa e, por vezes negativa, em que os conflitos latentes podiam rapidamente se converter em agressões verbais ou mesmo físicas. A capacidade assistencial, em consequência dessas limitações operacionais, apresentava grande fragilidade, mesmo no desempenho de suas atividades mais básicas - um cenário que compromete tanto os resultados em saúde quanto o próprio estabelecimento de indicadores de produção e gestão clínico-assistencial.

\section{Discussão}

A experiência etnográfica, ao descortinar o fato e as expectativas sobre a atenção farmacêutica, 
revelou que esse exercício profissional ainda não consolidou o paradigma proposto por Hepler e Strand $\mathbf{4}^{\mathbf{4}}$, e compartilhado pela política farmacêutica nacional ${ }^{7}$, de uma prática centrada no paciente (patient-oriented practice). Identificou-se que ainda pairam sobre os farmacêuticos ambiguidades, que são incompatíveis com um conceito amadurecido, no qual os paradigmas podem ser identificados com relativa facilidade.

Para Kuhn ${ }^{8}$, enquanto o mapa paradigmático ainda estiver se mostrando frutífero e não surgirem embaraços sérios no ajuste empírico da teoria, é mantido um comprometimento tenaz ao paradigma em vigência. Portanto, os resultados apresentados sugerem que ainda se está na fase de penumbra ou 'crise paradigmática', na qual o antigo paradigma ainda não foi totalmente abandonado ante a superioridade do novo. Isso se observa nos discursos que sobrepõem os conceitos de atenção farmacêutica e assistência farmacêutica; na rotina fortemente dirigida à logística e de distribuição de medicamentos e na dificuldade em definir os contornos e conteúdo da atenção farmacêutica.

Parece que os esforços políticos e acadêmicos para consolidação e normalização desse novo desenho de prática ainda não lograram êxito em solucionar o quebra-cabeças da ação farmacêutica. Por isso, diferentes desenhos se multiplicam, produzindo uma diversidade de configurações práticas e delineamentos dirigidos à atenção farmacêutica, assim como a sua própria nomeação (atenção farmacêutica ${ }^{6}$ ou cuidado farmacêutico ${ }^{7}$ ).

Ainda que os resultados apresentados não sejam capazes de generalização, uma rápida investigação da literatura nacional sugere que realmente se está em uma franca transição paradigmática. Mesmo quando os trabalhos científicos acenam para dispositivos de submissão ao paradigma da atenção farmacêutica, um olhar restrito à avaliação da terapêutica farmacológica prevalece diante da construção colaborativa do cuidado. Parece verossímil um esforço em superar a centralização exclusiva ao medicamento, mas ainda se observa uma fraca adesão, tanto aos princípios que organizam o campo assistencial no sistema público de saúde ${ }^{11}$ quanto a uma concepção emancipatória do cuidado.

Para Araújo e colaboradores ${ }^{3(2)}$, sobre a prática da atenção farmacêutica, ainda permanecem algumas dificuldades à constituição de seu significado. Segundo os autores:

[...] em todo o mundo, a definição adotada por Hepler e Strand (1990) é considerada como um marco consensual, mas no Brasil, o que se denomina como atenção farmacêutica assume significados distintos no que denominaríamos de 'mundo da ciência' e o como as políticas no âmbito da assistência farmacêutica a compreendem. As diferenças operam no plano epistemológico e se reproduzem na prática.

Para Kuhn ${ }^{8}$, os fundamentos de um paradigma são fornecidos a partir das atividades da comunidade científica, e para a consolidação da transição para a maturidade e normalidade cientifica, é exigido o reconhecimento de uma realização exemplar (no caso da atenção farmacêutica, o reordenamento do cuidado para centrado no paciente). Desse modo, pode-se constatar que o processo de alfabetização científica e tecnológica ainda se encontra em andamento.

Para Björkman ${ }^{12}$, há duas ideologias dominantes, que dirigem o contexto da atenção farmacêutica: uma centrada no paciente e outra convergindo para a medicina baseada em evidências. Segundo os autores, "different forms of pharmaceutical care have been developed depending on what ideology the conception is based on and to what extent this ideology is set in focus 11(339)". A essa análise, Gastelurrutia ${ }^{\mathbf{3}}$ apresentou uma contestação, em que reivindicava ao método Dáder as disposições suficientes para combinar de maneira eficaz essas duas ideologias. No entanto, o que se observa dessa controvérsia é o equívoco em pensar que é o método que realiza a prática, e não as pessoas. Como demonstra Khun ${ }^{8}$, as ciências são construções humanas, portanto, 
sociais e históricas, e não se sustentam apenas por estruturas metodológicas - muito menos por simples proselitismo.

Se ainda é possível se mover sob o dogma do medicamento, é preciso identificar os comportamentos e atitudes que não permitem o avanço do novo paradigma. Ademais, no desvelamento entre o que a profissão farmacêutica é, aquilo que poderia (ou deveria) ser e para onde irá, abrigam-se as questões fundamentais, para a compreensão de suas práticas, venturas e desventuras. Todos esses elementos devem ser levados em consideração, enfatizando como esses valores se irradiam à atenção farmacêutica.

\section{Conclusões}

Assim, ao problematizar o assentamento do paradigma da atenção farmacêutica no cenário brasileiro, parece claro que ainda persiste uma situação de penumbra e status paradigmático duvidoso. Os resultados ora descritos permitem afirmar que os farmacêuticos ainda apresentam grande resistência em operar para além dos limites do controle dos medicamentos. Deste modo, se, como afirma Cordeiro ${ }^{14}$, a revolução da profissão farmacêutica atende pelo nome de atenção farmacêutica, segundo a esta análise, essa revolução ainda não aconteceu.

\section{Colaboradoras}

Penaforte T (0000-0003-3606-7453)* concebeu e delineou o trabalho; conduziu a análise, interpretação dos dados e redação do manuscrito. Castro S (0000-0002-0241-9564)* contribuiu para a coleta, organização, análise dos dados, participou da discussão dos resultados e aprovou a versão final do manuscrito.

\section{Referências}

1. Del Corral FSD, Souza MLA, Negrão OL. Do boticário ao farmacêutico: o ensino de Farmácia na Bahia, de 1815 a 1949. Salvador: EDUFBA; 2009.

2. Costa EA, Araújo OS, Penaforte TR, et al. Concepções de assistência farmacêutica na atenção primária à saúde, Brasil. Rev. Saúde Pública. 2017; 51(supl2):5.

3. Araújo PS, Costa EA, Guerra Junior AA, et al. Atividades farmacêuticas de natureza clínica na atenção básica no Brasil. Rev. Saúde Pública. 2017; 51(supl2):6s.
4. Hepler CD, Strand LM. Opportunities and responsibilities in pharmaceutical care. Am. J. Hosp. Pharm. 1990; 47(3):533-543.

5. Dáder MJF, Martínez-Martínez F. La Atención Farmacéutica en farmacia comunitaria: evolución de conceptos, necesidades de formatión, modalidades y estrategias para su puesta en marcha. Pharm. Care Esp. 1999; 1(1):52-61.

6. Brasil. Ministério da Saúde; Conselho Nacional de Saúde. Resolução n 338, de 6 de maio de 2004. Apro- 
var a Política Nacional de Assistência Farmacêutica. 2004 maio 6. [acesso em 2020 nov 24]. Disponível em: https://bvsms.saude.gov.br/bvs/saudelegis/ cns/2004/res0338_06_05_2004.html.

7. Brasil. Ministério da Saúde, Secretaria de Ciência, Tecnologia e Insumos Estratégico. Cuidado farmacêutico na atenção básica. Caderno 1: Serviços Farmacêuticos na atenção básica à saúde. Brasília: Ministério da saúde, 2015. 108 p.

8. Kuhn T. A estrutura das revoluções cientificas. São Paulo: Perspectiva; 1998.

9. Masterman M. A natureza de um paradigma. In: Lakatos I, Musgrave A, organizadores. A crítica e o desenvolvimento do conhecimento. São Paulo: Cultrix; 1979.

10. Gill R. Análise de Discurso. In: Bauer MW, Gaskell GG, editores. Pesquisa qualitativa com texto, imagem e som: um manual. Petrópolis: Vozes; 2002.
11. Barros DSL, Silva DLM, Leite SN. Serviços farmacêuticos clínicos na atenção primária à saúde do Brasil. Trab. educ. saúde. 2020; 18(1):1-17.

12. Björkman IK, Bernsten CB, Sanner MA. Care ideologies reflected in 4 conceptions of pharmaceutical care. Res Social Adm Pharm. 2008; (4):332-342.

13. Gastelurrutia MA, Faus MJ. Different ways of practicing pharmaceutical care. Res Social Adm Pharm. 2009; 5(1):1-2.

14. Cordeiro BC, Reynaud F. Atenção farmacêutica: evolução ou revolução? In: Cordeiro BC, Leite SN. O farmacêutico na atenção à saúde. Itajaí: Editora da Universidade do Vale do Itajaí; 2005.

Recebido em 12/01/2021

Aprovado em 18/09/2021

Conflito de interesses: inexistente

Suporte financeiro: não houve 